Published in final edited form as:

Surg Oncol Clin N Am. 2019 July ; 28(3): 337-351. doi:10.1016/j.soc.2019.02.003.

\title{
Vaccine Strategy in Melanoma
}

\author{
Minyoung Kwak, M.D., M.P.H. ${ }^{\star}, 1,2,4$, Katie M. Leick, M.D., M.S. ${ }^{*}, 1,3,4$, Marit M. Melssen, M.S. \\ 1,5,6, Craig L. Slingluff Jr., M.D. ${ }^{1,4}$
}

${ }^{1}$ Division of Surgical Oncology, Department of Surgery, University of Virginia, Charlottesville, VA, USA ${ }^{2}$ Department of Surgery, SUNY Downstate, Brooklyn, NY, USA ${ }^{3}$ Department of Surgery, University of lowa, lowa City, IA, USA ${ }^{4}$ Carter Immunology Center, University of Virginia, Charlottesville, VA, USA ${ }^{5}$ Department of Microbiology, Immunology, and Cancer Biology, University of Virginia, Charlottesville, VA, USA ${ }^{6}$ Department of Immunohematology and Blood Transfusion, Leiden University Medical Center, Leiden, The Netherlands

\section{SYNOPSIS}

The incidence of melanoma continues to increase even as advances in immunotherapy have led to survival benefits in advanced stages. Vaccines are capable of inducing strong, anti-tumor immune responses with limited toxicity. Some vaccines have demonstrated clinical benefit in clinical trials alone; however, others have not despite inducing strong immune responses. Recent advancements have improved vaccine design, while combining vaccines with other immunotherapies offers promise. This review highlights the underlying principles of vaccine development, common components of vaccines, the remaining challenges and future directions of vaccine therapy in melanoma.

\section{Keywords}

Melanoma; Vaccine; Tumor-Associated Antigen; Neoantigen; Vaccine Adjuvant; T cell

\section{Introduction}

The development of vaccines against cancer has driven significant advancements in the field of tumor immunology, leading to a better understanding of the immune response against cancer. There is new expertise on the nature of tumor-associated antigens, making it possible to even genetically engineer immunogenic antigens for melanoma vaccines. This has been

CORRESPONDING AUTHOR Craig L. Slingluff, Jr., M.D. Address: P.O. Box 800709, Charlottesville, VA, 22908-0709, USA, clssh@virginia.edu.

Minyoung Kwak and Katie Leick contributed equally to this manuscript and are considered co-first authors. DISCLOSURE STATEMENT

Minyoung Kwak, Katie Leick, and Marit Melssen: nothing to disclose

Craig Slingluff, Jr: see chart below

Publisher's Disclaimer: This is a PDF file of an unedited manuscript that has been accepted for publication. As a service to our customers we are providing this early version of the manuscript. The manuscript will undergo copyediting, typesetting, and review of the resulting proof before it is published in its final form. Please note that during the production process errors may be discovered which could affect the content, and all legal disclaimers that apply to the journal pertain. 
achieved through advances in sequencing and manipulating genomes, development of algorithms to define putative $\mathrm{T}$ cell antigens, identification of different immune cell types, and unraveling complexities of the tumor microenvironment.

The promise of cancer vaccines is that vaccines induce targeted, tumor-specific immune responses with long-term memory in cases of recurrence or metastasis, with low risk of toxicity overall. There has been renewed focus on the potential of immunotherapy due to the recent success of checkpoint blockade therapy in advanced melanoma. When patients lack a pre-existing immune response to their cancer, they are unlikely to respond to checkpoint blockade; so, there is renewed interest in vaccines to induce antitumor immune responses that did not arise spontaneously. There is potential value of combining vaccine therapy with other immunotherapies to improve tumor control. Clinical trials implementing these combination therapies are currently underway. In this review, we summarize the current antigen and vaccine adjuvant strategies under investigation, and highlight the progress made with recent melanoma vaccine therapies in clinical trials.

\section{Background on Tumor Antigenicity and Immune Activation}

Cancer vaccines contain tumor-associated antigens and vaccine adjuvants to elicit the activation of dendritic cells and antigen-specific $\mathrm{T}$ cells. Initiation of the immune response against tumor cells occurs through recognition of tumor-associated antigens that must be processed and presented on MHC complexes by antigen-presenting cells (APCs) (Figure 1). Specific $\mathrm{T}$ cells recognize these MHC-antigen complexes leading to their activation and proliferation. Antigen presentation on MHC class I generally activates cytotoxic CD8 T cells $\left(\mathrm{T}_{\mathrm{CD} 8}\right)$, whereas presentation on MHC class II activates CD4 helper $\mathrm{T}$ cells $\left(\mathrm{T}_{\mathrm{CD} 4}\right)$. Elements of both CD8 and CD4 T cells are likely needed to mount an optimal response for tumor control and long-term memory.

Cancer vaccine formulations also incorporate 'vaccine adjuvants' to increase $\mathrm{T}$ cell stimulation by activating APCs, thereby enhancing antigen presentation and co-stimulation. Many vaccine adjuvants stimulate pattern-recognition receptors (PRRs) on APCs. These PRRs recognize PAMPs (pathogen- associated molecular patterns) or DAMPs (damageassociated molecular patterns), heat shock proteins, or reactive oxygen intermediates ${ }^{1}$. PAMPs include Toll-like receptor (TLR) agonists, such as $\mathrm{CpG}$ sequences. Introducing a tumor-associated antigen with vaccine adjuvants allows for an improved antigen-specific immune response to enhance tumor control.

\section{Vaccine Strategies: Antigen}

Antigens used in melanoma vaccines may be shared across patients or may be neoantigens that are uniquely expressed. Different types of antigens have been identified in melanoma (Figure 2), and are summarized below. The type of antigen that is selected can determine the tumor-specificity, type, and strength of the ensuing immune response.

\section{Shared melanoma antigens}

Melanocytic differentiation antigens-Melanocytic differentiation antigens are expressed by most melanoma tumors and induce $\mathrm{T}$ cell responses ${ }^{2,3}$. The shared aspect of 
these antigens enables broad application and generalization of shared antigen-targeting vaccines across multiple patients. Tyrosinase, TRP-2, Melan-A/MART-1, and gp-100 are common source proteins that are also expressed on normal melanocytes and on a few other pigmented cells. Thus, successful immune targeting of melanocytic antigens has the potential to induce autoimmunity to melanocytes. Additionally, since these antigens are present in some normal tissues, responses to them may be limited by pre-existing central tolerance ${ }^{4}$. Regardless, some vaccine and adoptive therapies targeting these antigens have been successful ${ }^{5,6}$.

Shared mutated antigens-Mutated antigens arise from acquired somatic mutations or single-nucleotide polymorphisms (SNPs) in melanoma cells and are often unique to a given patient tumor, although some are present in a fraction of patients. Because mutated antigens arise through tumorigenesis, they are absent in normal cells. BRAF, KIT, and NRAS mutations are common mutated antigens in melanoma. Antigenic BRAF peptides encompassing the $\mathrm{V} 600 \mathrm{E}$ driver mutation of melanoma have been reported ${ }^{7-10}$, inducing BRAF-specific immune responses in humans ${ }^{9,10}$ and tumor control in mice ${ }^{7,8}$. These data support further clinical investigation of these mutated antigens in vaccines.

Cancer germ line antigens-Cancer germ line antigens are expressed in the placenta or testis as immune privileged sites ${ }^{11}$, while also being uniquely expressed in some malignant tumors. The immunogenicity and unique expression of these antigens in cancers, rather than in normal cells, provides an opportunity to elicit an antitumor immune response using vaccines. Their restricted expression justifies considering them a form of neoantigen.

MAGE-A1, MAGE-A3, BAGE, GAGE, and NY-ESO-1 are a few examples of cancer germ line antigens that have been identified. Adoptive $\mathrm{T}$ cell therapy using $\mathrm{T}$ cells expressing a $\mathrm{T}$ cell receptor (TCR) transduced with NY-ESO-1 induced objective clinical responses in 55\% of patients with advanced melanoma ${ }^{12}$. Thus, cancer germ line antigens can be effective tumor regression antigens. On the other hand, a MAGE-A3 vaccine failed to enhance survival in a phase III trial ${ }^{13}$; so, the use of cancer germ line antigens in vaccines remains to be optimized. Although some cancer germ line antigens are solely expressed on tumor cells, a few studies using adoptive $\mathrm{T}$ cell therapies have shown cross-reactivity with normal tissues when very high affinity TCRs are used ${ }^{14}$. Interestingly, some cancer germ line antigens may be sequestered and thus not subject to preexisting tolerance, whereas others may not be sequestered ${ }^{15}$. Understanding this phenomenon may improve selection of cancer germ line antigens for cancer vaccines.

Phosphopeptides-Phosphorylation of oncogenic proteins supports malignant transformation; thus, targeting them is a promising strategy. Phosphorylated peptides derived from those proteins can be presented by both MHC class I and II molecules, which induces an immune response to the phosphorylated peptide sequence specifically ${ }^{16-19}$. Identification of tumor-specific phosphopeptide antigens may provide opportunities for personalized immune vaccine therapies ${ }^{20}$. A first-in-humans clinical trial has recently been completed (NCT01846143). 
Mutated neoantigens-The term 'neo-antigen' refers to newly expressed or acquired antigens, as in genomic mutations found within tumors but not in normal somatic cells. Due to its unique genetic sequence, the transcribed DNA, RNA, or translated peptide fragments can be used as a unique source of tumor-associated antigens. The advantage of these mutated neoantigens in vaccines is that it may avoid pre-existing central tolerance that is expected with shared antigens ${ }^{4}$ and should reduce risks of on-target autoimmune reactivity. Data also suggests that therapeutic $\mathrm{T}$ cells may respond more strongly to mutated neoantigens than to shared antigens ${ }^{21,22}$. Newer approaches have developed algorithms to predict and select advantageous immunogenic features of the mutanome. These mutated epitopes can be engineered into vaccines using a personalized approach ${ }^{23}$. A disadvantage of this personalized technique is the time required for the synthesis of personalized vaccines. Methods to predict immunogenic neoantigens were applied to patients and successfully showed immunogenicity of predicted neoantigens with promising clinical outcomes in a small number of patients ${ }^{23-25}$. However, much research still needs to be done to expand these preliminary results and to optimize immune responses to neoantigens.

\section{Antigen Type and Adjuvants}

Selection of an appropriate antigen type can influence the immune response following treatment. Vaccines may use antigen as whole tumor cells, RNA or DNA, single or multiple peptides, or APCs displaying the target antigen. Both efficacy and toxicity can be related to intrinsic immunologic potency, cross-reactivity of vaccine targets to antigen on normal cells, and associated adjuvants.

Peptide Vaccines-Peptide vaccines can be synthesized as short or long with single- or multi-peptide mixtures. Peptides are weakly immunogenic when naked peptide is used ${ }^{26,27}$; however, their use in combination with vaccine adjuvants or immune therapies induces potent, and frequently durable, $\mathrm{T}$ cell responses ${ }^{28-33}$.

Short Peptide Vaccines-Short peptide vaccines representing minimal CD8 epitopes (usually 9 amino acids long) thus elicit a cytotoxic $\mathrm{T}_{\mathrm{CD} 8}$ response by binding MHC class $\mathrm{I}$. Their effects on $\mathrm{T}_{\mathrm{CD} 8}$ are advantageous as it activates effector cells directly, thus negating the need for further antigen processing by APCs. Immune response rates may approach $100 \%$ in humans, of which $1-5 \%$ of the $\mathrm{T}_{\mathrm{CD} 8}$ population are peptide-specific effector $\mathrm{T}_{\mathrm{CD} 8}{ }^{28,29,31}$. Minimal epitope vaccines can induce strong $\mathrm{T}_{\mathrm{CD} 8}$ responses alone ${ }^{29}$. One study designed minimal epitope vaccines based on individual patient mutated neoantigens and generated neoantigen-specific $\mathrm{T}$ cell responses in all three patients, affecting 23-89\% of the $\mathrm{T}_{\mathrm{CD} 8}$ population; memory $\mathrm{T}$ cells were present for up to 4 months ${ }^{34,24,35}$. Short peptide vaccines may be limited by proteolytic degradation ${ }^{36}$, tolerance due to suboptimal antigen presentation, and less sustained immune responses ${ }^{37}$.

Extensive work has been done with a modified gp100 peptide (gp100 209-217;209-2M) that is a modified form of peptide naturally presented by HLA-A2 to $\mathrm{T}_{\mathrm{CD} 8}$. Vaccination with gp100 209-2M alone increased peptide-specific T cells in 97\% of patients ${ }^{31}$. Addition of gp100 to IL-2 in a clinical trial involving patients with advanced melanoma significantly increased clinical response rates and improved progression-free survival ${ }^{38,39}$. However, 
combination of gp100 peptide with checkpoint blockade therapies such as ipilimumab showed no therapeutic benefit compared to ipilimumab alone ${ }^{40}$. Mechanisms underlying this limitation have been explored in mice and suggest the addition of gp100 peptide vaccine in an antigen-depot vaccine with ipilimumab may lead to sequestration and destruction of nongp100-specific effector $\mathrm{T}$ cells, particularly those effector $\mathrm{T}$ cells that are induced by ipilimumab $^{41}$. This finding needs to be studied further in patients.

Helper Peptide Vaccines-Helper $\mathrm{T}_{\mathrm{CD} 4}$ are activated by recognition of peptide presented on class II MHC molecules and induce a multifaceted immune response by supporting $\mathrm{APC}, \mathrm{T}_{\mathrm{CD} 8}$ effector function, and $\mathrm{T}$ cell memory formation. Thus, immune therapies targeting helper $\mathrm{T}_{\mathrm{CD} 4}$ offer promise for tumor control ${ }^{32}$. A peptide vaccine composed of a mixture of 6 melanoma helper peptides (6MHP) that are recognized by $\mathrm{T}_{\mathrm{CD} 4}$ has been used in clinical trials and induces a Th1 $\mathrm{T}_{\mathrm{CD} 4}$ response without increasing the proportion of $\mathrm{T}_{\text {regs }}$ (regulatory $\mathrm{T}$ cells). $\mathrm{T}_{\mathrm{CD} 8}$ responses also occurred via epitope spreading ${ }^{27}$. Sixty-five percent of 6MHP- treated patients with stage IV melanoma developed an immune response, and treatment with vaccine significantly improved overall survival compared to matched controls ${ }^{27}$. Patient survival was associated with antibody response rates and the formation of memory $\mathrm{T}_{\mathrm{CD} 4}$ immune responses ${ }^{26}$.

Long Peptide Vaccines-Long peptide vaccines refer to lengths of 20-30 amino acids and carry the potential benefit of activating both $\mathrm{T}_{\mathrm{CD} 8}$ and $\mathrm{T}_{\mathrm{CD} 4}$ responses. Murine work demonstrated more efficient internalization and processing of long peptides by APCs compared to protein, resulting in more sustained $\mathrm{T}_{\mathrm{CD} 8}$ activation ${ }^{42}$. A phase I clinical trial of NY-ESO-1-derived long peptide (30 amino acids) combined with adjuvant induced antigenspecific $\mathrm{T}$ cells only when peptide was used in combination with adjuvant, but not with peptide alone ${ }^{32}$. Vaccination with long peptide targeting up to 20 predicted personal neoantigens successfully induced neoantigen-specific $\mathrm{T}_{\mathrm{CD} 4}$ responses to $60 \%$ of antigens, while $\mathrm{T}_{\mathrm{CD} 8}$ responses were limited to only $16 \%$ of unique neoantigens, suggesting preferential activation of $\mathrm{T}_{\mathrm{CD} 4}$ by long peptides ${ }^{25}$. Additionally, 4 of 6 patients had no recurrence 25 months after vaccination, suggesting the potential for clinical benefits and long-lasting effects of long peptide vaccines ${ }^{25}$.

RNA, DNA, and Protein Vaccines-RNA or DNA encoding genes for tumor antigens or immune enhancers can be introduced into APCs or myocytes through bacterial or viral vectors to synthesize peptides and mediate a vaccine effect. Some RNA vaccines involve electroporation of APCs to enable incorporation of mRNA encoding melanoma- associated antigens or immunostimulatory ligands to facilitate antigen-specific $\mathrm{T}$ cell responses. In such studies, $\mathrm{T}_{\mathrm{CD} 8}$ responses were detected in $57-80 \%$ of patients, but no objective clinical responses were observed ${ }^{43,44}$. Some recent RNA vaccines are designed and personalized based on mutations expressed and identified by RNA sequencing and selected for predicted high affinity binding to MHC class I and II. These are engineered into synthetic RNA and delivered using a vaccine vehicle. The majority of responses induced were $\mathrm{T}_{\mathrm{CD} 4}$ responses $^{23}$, comparable to personalized neoepitope vaccines based on mutated peptides ${ }^{25}$. Neoepitope vaccination also resulted in a broadened repertoire of $\mathrm{T}$ cells ${ }^{23}$. A phase I clinical trial of DNA vaccine-encoding genes for immunogenic epitopes for gp100 and 
TRP2 demonstrated an immune response rate of $84 \%$, comparable to other peptide vaccines using gp $100^{45,38}$. Despite induction of an immune response, DNA vaccines evoke limited objective clinical responses ${ }^{45}$.

Whole Cell Vaccines-Whole cancer cells can be integrated into vaccines and serve as a source of antigen for APC presentation. They contain numerous mutated neoantigens that are inherent to the tumor, which does not mandate that they be identified prior to designing and manufacturing the vaccine. Whole cells can be modified to express particular tumor antigens or immune enhancers to further potentiate immune responses. This type of vaccine is typically more proficient at inducing expansion of $\mathrm{T}_{\mathrm{CD} 4}$ than $\mathrm{T}_{\mathrm{CD} 8}$, resulting in an attenuated antitumor immune response. Despite whole cell vaccines showing initial promise to prolong survival with high clinical response rates, a large randomized phase III clinical trial showed no significant clinical benefit ${ }^{46}$. There is currently a phase 3 clinical trial underway (NCT01546571) using a cell-based vaccine derived from cell line supernatants containing antigens shed by tumor cells, which significantly improved disease-free survival in vaccine-treated patients in a prior phase 2 trial $^{47}$.

\section{Vaccine Strategies: Vaccine Adjuvants}

Vaccine adjuvants are aimed at producing more robust immune responses by increasing antigen uptake and presentation, recruiting other immune cells, and/or forming a depot effect for sustained release of antigen ${ }^{48,49}$.

\section{Incomplete Freund's Adjuvant (IFA)}

A common adjuvant used in peptide vaccines for melanoma is Montanide ISA 51 (Seppic, Inc), a form of Incomplete Freund's Adjuvant (IFA). This is an oil-based agent where droplets of aqueous peptide are contained within a surrounding oil phase. This facilitates a depot effect at the vaccine site, allowing for continued antigen exposure in a stabilized oil emulsion ${ }^{48}$. In humans, immune responses to peptides in IFA were greater than peptides pulsed on APCs ${ }^{50,51}$. In mice, the depot effect of IFA may also lead to vaccine-site sequestration of activated $\mathrm{T}$ cells against antigen ${ }^{52}$ that may prevent $\mathrm{T}$ cell homing to tumor. There are data suggesting the added immunologic benefit of other adjuvants such as CD40 stimulating antibody and TLR agonists instead of IFA to avoid these concerns. Human studies have not yet been done to determine whether CD40 antibody plus TLR agonists can induce a much stronger $\mathrm{T}$ cell response than IFA alone; however, our studies continue to support the addition of IFA to TLR agonists in melanoma vaccines with short peptides ${ }^{53}$.

\section{Dendritic Cell Vaccines}

Dendritic cell (DC) vaccines use autologous DC to present antigen and to stimulate immune cells by releasing pro-inflammatory cytokines. In preparation for vaccine synthesis, DCs are isolated from peripheral blood. The vaccine can then be packaged using various adjuvants to induce DC activation, or autologous DCs can be pulsed with antigen ex-vivo and administered in the same patient. Vaccination route may determine the tissue site where the DCs will migrate ${ }^{54}$. A randomized phase II trial using autologous DC vaccines with GMCSF in patients with advanced melanoma showed longer survival compared to a tumor cell 
vaccine, with $70 \%$ reduction in risk of death ${ }^{55}$. However, this trial had low patient numbers and unequal prognostic factors. Other trials demonstrated lower $\mathrm{T}$ cell responses with DC vaccines when compared to peptide vaccines with adjuvant ${ }^{50,51}$.

Another potential method of further stimulating DCs in murine models is an in-vivo method using viral vectors and CD40 stimulating antibodies. CD40 is a co-stimulatory receptor on DCs that adds to $\mathrm{DC}$ maturation and $\mathrm{T}_{\mathrm{CD} 8}$ activation ${ }^{56,57}$. The addition of CD40 antibody to a combination of TLR3 agonists and a neoantigen vaccine against colon adenocarcinoma in mice showed improved survival compared to either agonist alone, with expansion of neoantigen-specific $\mathrm{T}$ cells in both the periphery and in the tumor microenvironment ${ }^{58}$. These preclinical results show promise in the strength of CD40 for DC activation but still need to be evaluated in humans.

Toll-like Receptor (TLR) Agonists-TLR agonists stimulate PRRs on DCs to promote antigen processing and presentation to T cells. A common TLR agonist used in melanoma vaccine adjuvants is the TLR3 agonist poly-ICLC (polyinosine-polycytidylic acid). This agonist matures DCs to generate $\mathrm{T}_{\mathrm{CD} 8}$ and NK cells with higher cytotoxic capacity ${ }^{59,60}$. The combination of IFA and poly-ICLC with long NY-ESO-1 peptides showed that both adjuvants had different but beneficial effects on antigen-specific activation of $\mathrm{T}_{\mathrm{CD} 4}$ : emulsification of the antigen in IFA increased antigen-specific $\mathrm{T}_{\mathrm{CD} 4}$, while poly-ICLC induced a Th1 phenotype, leading to increased cell-mediated immunity and inflammatory response $^{61}$.

$\mathrm{CpG}$ is an oligodeoxynucleotide fragment rich in cytosine and guanine, similar to bacterial DNA that is an agonist for TLR9 and induces DC maturation ${ }^{62,63}$. With DC maturation, $\mathrm{CpG}$ has also been shown to increase co-stimulatory surface marker expression with increases in proinflammatory cytokines needed for cytotoxic $\mathrm{T}_{\mathrm{CD} 8}$ co-stimulation ${ }^{62,63}$, and possibly B cell stimulation ${ }^{64}$. Addition of $\mathrm{CpG}$ to IFA has dramatically enhanced $\mathrm{T}$ cell responses to a short peptide vaccine ${ }^{62}$. Despite its effectiveness in $\mathrm{T}_{\mathrm{CD} 8}$ promotion, a mouse model suggests it may require decreased levels of immunosuppressive $T_{\text {regs }}$ and multiple booster vaccinations to maximize its effect ${ }^{65}$. On the other hand, resiquimod (R848) is an agonist for TLR7 and TLR8 that has been found to decrease the immunosuppressive function of $\mathrm{T}_{\text {regs }}{ }^{66}$ and myeloid-derived suppressor cells (MDSC) ${ }^{67}$, supporting increased $\mathrm{T}$ cell proliferation.

\section{Systemic Cytokines}

Other types of vaccine adjuvants involve the use of immune cell cytokines. GM-CSF (granulocyte- macrophage colony-stimulating factor) is used to attract and activate DCs to further promote peptide antigen-specific responses ${ }^{68,69}$. GM-CSF enhanced immunogenicity of a cell-based vaccine in mice ${ }^{68}$, and combined with a peptide vaccine, increased $\mathrm{T}$ cell responses when compared to DCs pulsed with the same peptide antigen ${ }^{51}$. However, in a large randomized clinical trial, the addition of GM-CSF to a peptide vaccine significantly decreased responses in both $\mathrm{T}_{\mathrm{CD} 8}$ and $\mathrm{T}_{\mathrm{CD} 4}$ compared to peptide vaccine alone ${ }^{70}$. Also, another large randomized clinical trial revealed that adding GM-CSF to a melanoma cell 
vaccine resulted in worse survival and early melanoma-related death ${ }^{71}$. The use of GM-CSF as a vaccine adjuvant is cautionary and requires further investigation.

Systemic IL-2 infusions have also been used as a vaccine adjuvant in melanoma due to its overall stimulatory effect on $\mathrm{T}$ cells, and its clinical activity as high-dose monotherapy. When I L-2 was combined with the peptide vaccine gp-100, clinical responses were significantly higher than with I L-2 alone, with associated longer progression-free survival ${ }^{72}$. Peptide-specific immunogenicity occurred in 19\% of patients who received both gp100 and I L-2, but this antigen specificity did not correlate with clinical response. Other studies have failed to show immunologic or clinical benefit of I L-2, GM-CSF, or interferon-alpha in combination with melanoma vaccines ${ }^{73,74}$.

\section{Current Melanoma Vaccine Trials}

There are many ongoing clinical trials studying the impacts of various antigen formats and adjuvants on immune responses and clinical outcomes (Figure 3A). Among these, DC and peptide vaccines predominate. Peptide vaccines use a variety of adjuvants, the most common of which is IFA (Figure 3B). DC vaccines, as an adjuvant on their own, do not frequently utilize additional adjuvants (Figure 3C). Combination therapy with vaccines provides an opportunity to target the immune system through another mechanism to augment anti-tumor effects and potentiate clinical benefits. The most common combination therapy for peptide vaccines is checkpoint blockade, while IL-2 is most common among combination therapies used with DC vaccines, though DC vaccines utilize more combination therapies overall (Figure 3B, C).

\section{Novel approaches}

\section{Prophylactic melanoma vaccine}

With the success of the generation of a prophylactic vaccine for virus-induced cervical cancer, the idea of prophylactic vaccines against melanoma regains interest. Preclinical in vivo studies have shown potential of this approach, regardless of whether a DC-, RNA-, whole cell- or peptide-based antigen vehicle was used ${ }^{75-78}$.

\section{Stem cell-based vaccines}

Induced pluripotent stem cells (Ipsc's) are immunogenic pluripotent stem-like cells that can be generated from a patient's cells. Their gene expression is similar to embryonic stem cells and includes expression of many tumor antigens. In preclinical models of melanoma and other solid tumors, immunization and therapeutic vaccination of iPSC's induced robust $\mathrm{T}_{\mathrm{CD} 4}$ and $\mathrm{T}_{\mathrm{CD} 8}$ responses, as well as a reduction in tumor burden ${ }^{79}$. Though these studies are still in the preclinical phase, they show potential for generating effective, personalized vaccines.

\section{Conclusions}

Melanoma vaccine research has shown the potential for vaccines to elicit antigen-specific $\mathrm{T}$ cells as well as provide some tumor control. Understanding the importance of specific immunologic effects of antigen vehicle and adjuvant is vital to the progression of vaccine 
strategy. As our knowledge base expands through preclinical studies and results from recent clinical trials are finalized, we will be able to better optimize our approach for sustained tumor control.

Current therapies that involve vaccines alone have limitations due to discordance between antigen-specific T cell expansion and tumor control. Despite cancer vaccines showing lasting immune response rates up to $100 \%{ }^{28,31}$, vaccines alone have led to clinical response rates below $10 \%$ in most studies ${ }^{80}$. This low clinical response may be due to insufficient $\mathrm{T}$ cell priming, poor homing to tumor, dysfunction of $\mathrm{T}$ cells in the tumor microenvironment, or progressive loss of function. Some of these may be due to defects in antigen presentation, or immunosuppressive changes in the tumor microenvironment that could lead to anergic $\mathrm{T}$ cells or interference with $\mathrm{T}$ cell homing to tumor ${ }^{81}$. The advancement of neoantigen technology, along with newer adjuvant strategies, are all promising additions to increase clinical response. There are other preclinical methods of inhibiting immunosuppressive effects currently under investigation, as well. Therefore, the combination of vaccines with other immunotherapies has clear theoretical advantages as we await results of ongoing clinical trials.

\section{Appendix}




\begin{tabular}{|c|c|c|c|c|c|c|c|c|c|c|c|c|c|}
\hline 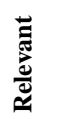 & $\stackrel{0}{\infty}$ &  & $\stackrel{0}{0}$ & $\stackrel{0}{0}$ & $\stackrel{0}{2}$ & z &  & 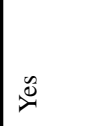 & 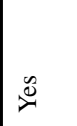 & 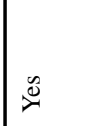 & z & 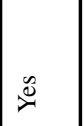 & $\stackrel{\mathscr{V}}{0}$ \\
\hline 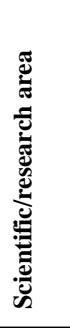 & 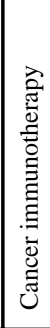 & 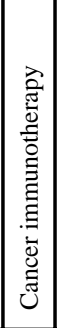 & 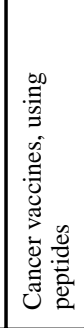 & 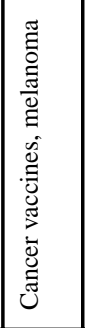 & 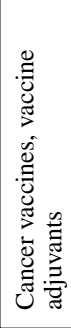 & 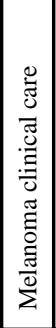 &  & 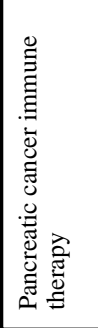 & 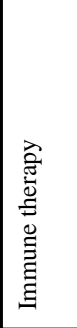 &  & 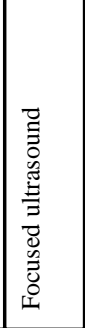 &  & 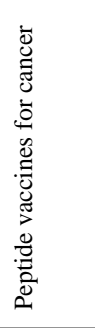 \\
\hline 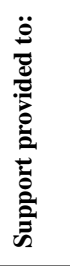 & 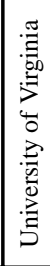 & 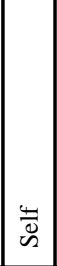 & $\mid \begin{array}{l}\text { w } \\
\text { s. }\end{array}$ & 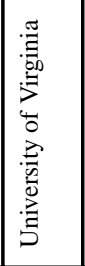 &  & 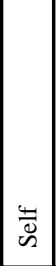 &  & 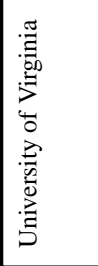 & 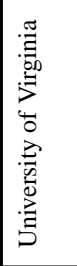 & 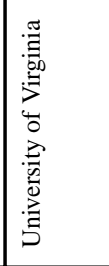 & 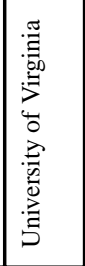 & 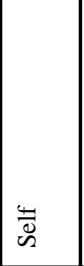 & 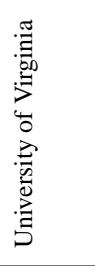 \\
\hline$\frac{\bar{z}}{3}$ & $\begin{array}{l}\infty \\
.0 \\
.0 \\
0 \\
0 \\
0\end{array}$ & 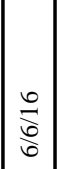 & 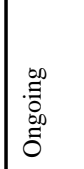 & 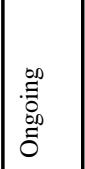 & 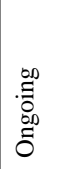 & 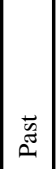 & 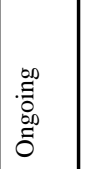 &  & 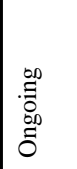 & 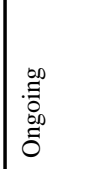 & 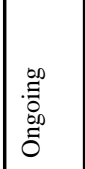 & 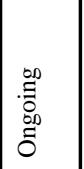 & 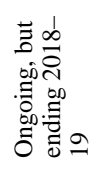 \\
\hline$\frac{\varrho}{2}$ & 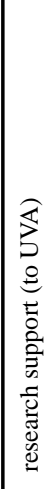 & 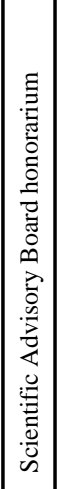 & 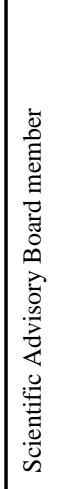 & 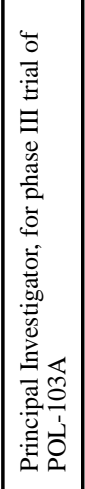 & 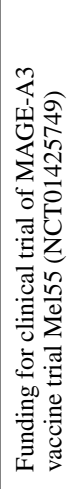 & 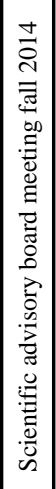 &  & 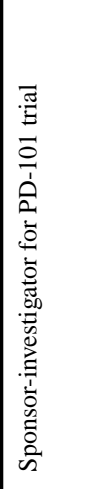 & 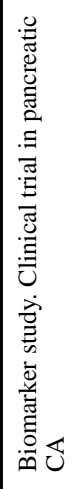 & 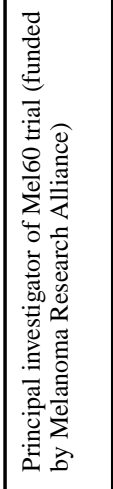 &  & 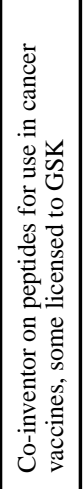 & 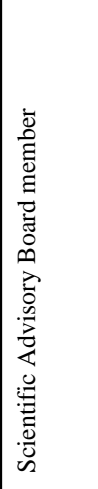 \\
\hline 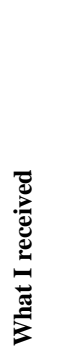 & 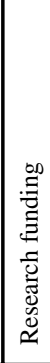 &  & 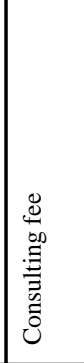 & 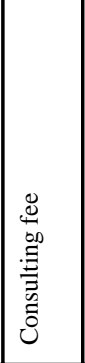 & 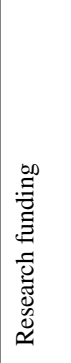 &  & 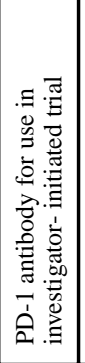 & 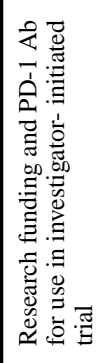 & 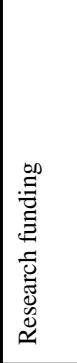 &  & 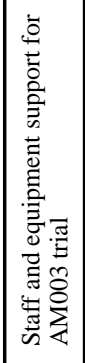 & 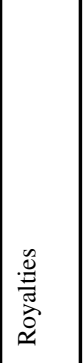 & 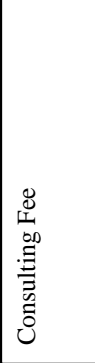 \\
\hline 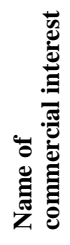 & $\frac{g}{g}$ & U. & 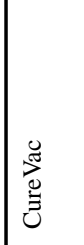 & 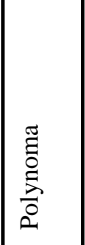 & 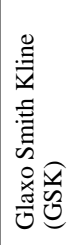 & 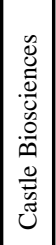 & 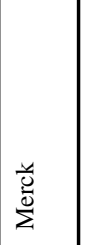 & 离 & 离 & m &  & 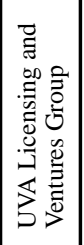 & 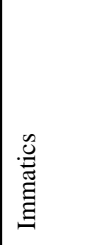 \\
\hline
\end{tabular}




\section{References}

1. Pradeu T, Cooper EL. The danger theory: 20 years later. Front Immunol. 2012;3:287. doi:10.3389/ fimmu.2012.00287 [PubMed: 23060876]

2. Bakker AB, Schreurs MW, de Boer AJ, et al. Melanocyte lineage-specific antigen gp100 is recognized by melanoma-derived tumor-infiltrating lymphocytes. J Exp Med. 1994;179(3):10051009. [PubMed: 8113668]

3. Reynolds SR, Celis E, Sette A, et al. HLA-Independent Heterogeneity of CD8+ T Cell Responses to MAGE-3, Melan-A/MART-1, gp100, Tyrosinase, MC1R, and TRP-2 in Vaccine-Treated Melanoma Patients. The Journal of Immunology. 1998;161(12):6970-6976. [PubMed: 9862732]

4. Colella TA, Bullock TNJ, Russell LB, et al. Self-Tolerance to the Murine Homologue of a Tyrosinase-Derived Melanoma Antigen: Implications for Tumor Immunotherapy. Journal of Experimental Medicine. 2000;191(7):1221-1232. doi:10.1084/jem.191.7.1221 [PubMed: 10748239]

5. Rosenberg SA, Yang JC, Schwartzentruber DJ, et al. Recombinant fowlpox viruses encoding the anchor-modified gp100 melanoma antigen can generate antitumor immune responses in patients with metastatic melanoma. Clin Cancer Res. 2003;9(8):2973-2980. [PubMed: 12912944]

6. Overwijk WW, Tsung A, Irvine KR, et al. gp100/pmel 17 is a murine tumor rejection antigen:induction of "self"-reactive, tumoricidal T cells using high-affinity, altered peptide ligand. J Exp Med. 1998;188(2):277-286. [PubMed: 9670040]

7. Liu Q, Zhu H, Liu Y, Musetti S, Huang L. BRAF peptide vaccine facilitates therapy of murine BRAF-mutant melanoma. Cancer Immunol Immunother. 2018;67(2):299-310. doi:10.1007/ s00262-017-2079-7 [PubMed: 29094184]

8. Cintolo JA, Datta J, Xu S, Gupta M, Somasundaram R, Czerniecki BJ. Type I-polarized BRAFpulsed dendritic cells induce antigen-specific CD8+ T cells that impact BRAF-mutant murine melanoma. Melanoma Res. 2016;26(1):1-11. doi:10.1097/CMR.0000000000000203 [PubMed: 26451873]

9. Somasundaram R, Swoboda R, Caputo L, et al. Human leukocyte antigen-A2-restricted CTL responses to mutated BRAF peptides in melanoma patients. Cancer Res. 2006;66(6):3287-3293. doi:10.1158/0008-5472.CAN-05-1932 [PubMed: 16540682]

10. Sharkey MS, Lizee G, Gonzales MI, Patel S, Topalian SL. CD4(+) T-cell recognition of mutated BRAF in melanoma patients harboring the V599E mutation. Cancer Res. 2004;64(5):1595-1599. [PubMed: 14996715]

11. Fijak M, Bhushan S, Meinhardt A. Immunoprivileged sites: the testis. Methods Mol Biol. 2011;677:459-470. doi:10.1007/978-1-60761-869-0_29 [PubMed: 20941627]

12. Robbins PF, Kassim SH, Tran TLN, et al. A pilot trial using lymphocytes genetically engineered with an NY-ESO-1-reactive T-cell receptor: long-term follow-up and correlates with response. Clin Cancer Res. 2015;21(5):1019-1027. doi:10.1158/1078-0432.CCR-14-2708 [PubMed: 25538264]

13. Dreno B, Thompson JF, Smithers BM, et al. MAGE-A3 immunotherapeutic as adjuvant therapy for patients with resected, MAGE-A3-positive, stage III melanoma (DERMA): a double-blind, randomised, placebo-controlled, phase 3 trial. The Lancet Oncology. 2018;19(7):916-929. doi: 10.1016/S1470-2045(18)30254-7 [PubMed: 29908991]

14. Johnson LA, Morgan RA, Dudley ME, et al. Gene therapy with human and mouse T-cell receptors mediates cancer regression and targets normal tissues expressing cognate antigen. Blood. 2009;114(3):535-546. doi:10.1182/blood-2009-03-211714 [PubMed: 19451549]

15. Tung KSK, Harakal J, Qiao H, et al. Egress of sperm autoantigen from seminiferous tubules maintains systemic tolerance. J Clin Invest. 2017;127(3):1046-1060. doi:10.1172/JCI89927 [PubMed: 28218625]

16. Petersen J, Wurzbacher SJ, Williamson NA, et al. Phosphorylated self-peptides alter human leukocyte antigen class I-restricted antigen presentation and generate tumor-specific epitopes. Proc Natl Acad Sci USA. 2009;106(8):2776-2781. doi:10.1073/pnas.0812901106 [PubMed: 19196958]

17. Mohammed F, Cobbold M, Zarling AL, et al. Phosphorylation-dependent interaction between antigenic peptides and MHC class I: a molecular basis for the presentation of transformed self. Nat Immunol. 2008;9(11):1236-1243. doi:10.1038/ni.1660 [PubMed: 18836451] 
18. Depontieu FR, Qian J, Zarling AL, et al. Identification of tumor-associated, MHC class IIrestricted phosphopeptides as targets for immunotherapy. Proc Natl Acad Sci USA. 2009;106(29): 12073-12078. doi:10.1073/pnas.0903852106 [PubMed: 19581576]

19. Mohammed F, Stones DH, Zarling AL, et al. The antigenic identity of human class I MHC phosphopeptides is critically dependent upon phosphorylation status. Oncotarget. 2017;8(33): 54160-54172. doi:10.18632/oncotarget.16952 [PubMed: 28903331]

20. Zarling AL, Obeng RC, Desch AN, et al. MHC-restricted phosphopeptides from insulin receptor substrate-2 and CDC25b offer broad-based immunotherapeutic agents for cancer. Cancer Res. 2014;74(23):6784-6795. doi:10.1158/0008-5472.CAN-14-0043 [PubMed: 25297629]

21. Robbins PF, Lu Y-C, El-Gamil M, et al. Mining exomic sequencing data to identify mutated antigens recognized by adoptively transferred tumor-reactive T cells. Nat Med. 2013;19(6):747752. [PubMed: 23644516]

22. Lu Y-C, Yao X, Crystal JS, et al. Efficient identification of mutated cancer antigens recognized by T cells associated with durable tumor regressions. Clin Cancer Res. 2014;20(13):3401-3410. doi: 10.1158/1078-0432.CCR-14-0433 [PubMed: 24987109]

23. Sahin U, Derhovanessian E, Miller M, et al. Personalized RNA mutanome vaccines mobilize poly specific therapeutic immunity against cancer. Nature. 2017;547(7662):222-226. doi:10.1038/ nature23003 [PubMed: 28678784]

24. Carreno BM, Magrini V, Becker-Hapak M, et al. A dendritic cell vaccine increases the breadth and diversity of melanoma neoantigen-specific T cells. Science. 2015;348(6236):803-808. doi: 10.1126/science.aaa3828 [PubMed: 25837513]

25. Ott PA, Hu Z, Keskin DB, et al. An immunogenic personal neoantigen vaccine for patients with melanoma. Nature. 2017;547(7662):217-221. doi:10.1038/nature22991 [PubMed: 28678778]

26. Reed CM, Cresce ND, Mauldin IS, Slingluff CL, Olson WC. Vaccination with Melanoma Helper Peptides Induces Antibody Responses Associated with Improved Overall Survival. Clin Cancer Res. 2015;21(17):3879-3887. doi:10.1158/1078-0432.CCR-15-0233 [PubMed: 25967144]

27. Hu Y, Kim H, Blackwell CM, Slingluff CL. Long-term outcomes of helper peptide vaccination for metastatic melanoma. Ann Surg. 2015;262(3):456-464; discussion 462-464. doi:10.1097/SLA. 0000000000001419 [PubMed: 26258314]

28. Slingluff CL, Petroni GR, Chianese-Bullock KA, et al. Immunologic and clinical outcomes of a randomized phase II trial of two multipeptide vaccines for melanoma in the adjuvant setting. Clin Cancer Res. 2007;13(21):6386-6395. doi:10.1158/1078-0432.CCR-07-0486 [PubMed: 17975151]

29. Slingluff CL, Petroni GR, Olson WC, et al. Effect of granulocyte/macrophage colony-stimulating factor on circulating CD8+ and CD4+ T-cell responses to a multipeptide melanoma vaccine: outcome of a multicenter randomized trial. Clin Cancer Res. 2009;15(22):7036-7044. doi: 10.1158/1078-0432.CCR-09-1544 [PubMed: 19903780]

30. Slingluff CL, Petroni GR, Chianese-Bullock KA, et al. Randomized multicenter trial of the effects of melanoma-associated helper peptides and cyclophosphamide on the immunogenicity of a multipeptide melanoma vaccine. J Clin Oncol. 2011;29(21):2924-2932. doi:10.1200/JCO. 2010.33.8053 [PubMed: 21690475]

31. Rosenberg SA, Sherry RM, Morton KE, et al. Tumor progression can occur despite the induction of very high levels of self/tumor antigen-specific CD8+ T cells in patients with melanoma. J Immunol. 2005;175(9):6169-6176. [PubMed: 16237114]

32. Sabbatini P, Tsuji T, Ferran L, et al. Phase I trial of overlapping long peptides from a tumor selfantigen and poly-ICLC shows rapid induction of integrated immune response in ovarian cancer patients. Clin Cancer Res. 2012;18(23):6497-6508. doi:10.1158/1078-0432.CCR-12-2189 [PubMed: 23032745]

33. Slingluff CL, Chianese-Bullock KA, Bullock TNJ, et al. Immunity to melanoma antigens: from self-tolerance to immunotherapy. Adv Immunol. 2006;90:243-295. doi:10.1016/ S0065-2776(06)90007-8 [PubMed: 16730266]

34. Nitschke NJ, Bjoern J, Iversen TZ, Andersen MH, Svane IM. Indoleamine 2,3-dioxygenase and survivin peptide vaccine combined with temozolomide in metastatic melanoma. Stem Cell Investig. 2017;4. doi:10.21037/sci.2017.08.06 
35. Zeng G, Li Y, El-Gamil M, et al. Generation of NY-ESO-1-specific CD4+ and CD8+ T Cells by a Single Peptide with Dual MHC Class I and Class II Specificities: A New Strategy for Vaccine Design. Cancer Res. 2002;62(13):3630-3635. [PubMed: 12097265]

36. Brinckerhoff LH, Kalashnikov VV, Thompson LW, et al. Terminal modifications inhibit proteolytic degradation of an immunogenic MART-1(27-35) peptide: implications for peptide vaccines. Int J Cancer. 1999;83(3):326-334. [PubMed: 10495424]

37. Knutson KL, Schiffman K, Cheever MA, Disis ML. Immunization of cancer patients with a HER-2/neu, HLA-A2 peptide, p369-377, results in short-lived peptide-specific immunity. Clin Cancer Res. 2002;8(5):1014-1018.

38. Schwartzentruber DJ, Lawson DH, Richards JM, et al. gp100 peptide vaccine and interleukin-2 in patients with advanced melanoma. N Engl J Med. 2011;364(22):2119-2127. doi:10.1056/ NEJMoa1012863 [PubMed: 21631324]

39. Kawakami Y, Eliyahu S, Jennings C, et al. Recognition of multiple epitopes in the human melanoma antigen gp100 by tumor-infiltrating T lymphocytes associated with in vivo tumor regression. J Immunol. 1995;154(8):3961-3968. [PubMed: 7706734]

40. Hodi FS, O'Day SJ, McDermott DF, et al. Improved Survival with Ipilimumab in Patients with Metastatic Melanoma. N Engl J Med. 2010;363(8):711-723. doi:10.1056/NEJMoa1003466 [PubMed: 20525992]

41. Hailemichael Y, Woods A, Fu T, et al. Cancer vaccine formulation dictates synergy with CTLA-4 and PD-L1 checkpoint blockade therapy. J Clin Invest. 128(4):1338-1354. doi:10.1172/JCI93303

42. Bijker MS, van den Eeden SJF, Franken KL, Melief CJM, Offringa R, van der Burg SH. CD8+ CTL priming by exact peptide epitopes in incomplete Freund's adjuvant induces a vanishing CTL response, whereas long peptides induce sustained CTL reactivity. J Immunol. 2007;179(8):50335040. [PubMed: 17911588]

43. Wilgenhof S, Van Nuffel AMT, Corthals J, et al. Therapeutic vaccination with an autologous mRNA electroporated dendritic cell vaccine in patients with advanced melanoma. J Immunother. 2011;34(5):448-456. doi:10.1097/CJI.0b013e31821dcb31 [PubMed: 21577140]

44. Wilgenhof S, Van Nuffel AMT, Benteyn D, et al. A phase IB study on intravenous synthetic mRNA electroporated dendritic cell immunotherapy in pretreated advanced melanoma patients. Ann Oncol. 2013;24(10):2686-2693. doi:10.1093/annonc/mdt245 [PubMed: 23904461]

45. Patel PM, Ottensmeier CH, Mulatero C, et al. Targeting gp100 and TRP-2 with a DNA vaccine: Incorporating $\mathrm{T}$ cell epitopes with a human $\mathrm{IgG} 1$ antibody induces potent $\mathrm{T}$ cell responses that are associated with favourable clinical outcome in a phase I/II trial. Oncoimmunology. 2018;7(6):e1433516. doi:10.1080/2162402X.2018.1433516

46. Faries MB, Mozzillo N, Kashani-Sabet M, et al. Long-Term Survival after Complete Surgical Resection and Adjuvant Immunotherapy for Distant Melanoma Metastases. Ann Surg Oncol. 2017;24(13):3991-4000. doi:10.1245/s10434-017-6072-3 [PubMed: 29019177]

47. Bystryn JC, Zeleniuch-Jacquotte A, Oratz R, Shapiro RL, Harris MN, Roses DF. Double-blind trial of a polyvalent, shed-antigen, melanoma vaccine. Clin Cancer Res. 2001;7(7):1882-1887. [PubMed: 11448900]

48. Awate S, Babiuk LA, Mutwiri G. Mechanisms of Action of Adjuvants. Front Immunol. 2013;4. doi:10.3389/fimmu.2013.00114 [PubMed: 23386848]

49. Temizoz B, Kuroda E, Ishii KJ. Vaccine adjuvants as potential cancer immunotherapeutics. Int Immunol. 2016;28(7):329-338. doi:10.1093/intimm/dxw015 [PubMed: 27006304]

50. O'Neill DW, Adams S, Goldberg JD, et al. Comparison of the immunogenicity of Montanide ISA 51 adjuvant and cytokine-matured dendritic cells in a randomized controlled clinical trial of melanoma vaccines. JCO. 2009;27(15S):3002-3002. doi:10.1200/jco.2009.27.15s.3002

51. Slingluff CL, Petroni GR, Yamshchikov GV, et al. Clinical and immunologic results of a randomized phase II trial of vaccination using four melanoma peptides either administered in granulocyte- macrophage colony-stimulating factor in adjuvant or pulsed on dendritic cells. J Clin Oncol. 2003;21(21):4016-4026. doi:10.1200/jc0.2003.10.005 [PubMed: 14581425]

52. Hailemichael Y, Dai Z, Jaffarzad N, et al. Persistent antigen at vaccination sites induces tumorspecific $\mathrm{CD}^{+}$T cell sequestration, dysfunction and deletion. Nat Med. 2013;19(4):465-472. doi: 10.1038/nm.3105 [PubMed: 23455713] 
53. Melssen M, Petroni G, Grosh WW, et al. A multipeptide vaccine plus toll-like receptor (TLR) agonists LPS or polylCLC in combination with incomplete Freund's adjuvant (IFA) in melanoma patients. 31st Annual Meeting and Associated Programs of the Society for Immunotherapy of Cancer (SITC 2016) 2016;4.

54. Mullins DW, Sheasley SL, Ream RM, Bullock TNJ, Fu Y-X, Engelhard VH. Route of Immunization with Peptide-pulsed Dendritic Cells Controls the Distribution of Memory and Effector T Cells in Lymphoid Tissues and Determines the Pattern of Regional Tumor Control. J Exp Med. 2003;198(7):1023-1034. doi:10.1084/jem.20021348 [PubMed: 14530375]

55. Dillman RO, Cornforth AN, Nistor GI, McClay EF, Amatruda TT, Depriest C. Randomized phase II trial of autologous dendritic cell vaccines versus autologous tumor cell vaccines in metastatic melanoma: 5-year follow up and additional analyses. J Immunother Cancer. 2018;6(1):19. doi: 10.1186/s40425-018-0330-1 [PubMed: 29510745]

56. Tacken PJ, de Vries IJM, Torensma R, Figdor CG. Dendritic-cell immunotherapy: from ex vivo loading to in vivo targeting. Nature Reviews Immunology. 2007;7(10):790-802. doi:10.1038/ nri2 2173

57. Hangalapura BN, Timares L, Oosterhoff D, Scheper RJ, Curiel DT, de Gruijl TD. CD40-targeted adenoviral cancer vaccines: the long and winding road to the clinic. J Gene Med. 2012;14(6):416427. doi:10.1002/jgm.1648 [PubMed: 22228547]

58. Hoki T, Yamauchi T, Odunsi K, Ito F. Synergistic anti-tumor efficacy of combined TLR3 and CD40 neoantigen vaccine requires Batf3-dependent dendritic cells. The Journal of Immunology. 2018;200(1 Supplement):181.7-181.7.

59. Alexopoulou L, Holt AC, Medzhitov R, Flavell RA. Recognition of double-stranded RNA and activation of NF-kappaB by Toll-like receptor 3. Nature. 2001;413(6857):732-738. doi: 10.1038/35099560 [PubMed: 11607032]

60. Perrot I, Deauvieau F, Massacrier C, et al. TLR3 and Rig-like receptor on myeloid dendritic cells and Rig-like receptor on human NK cells are both mandatory for production of IFN-gamma in response to double-stranded RNA. J Immunol. 2010;185(4):2080-2088. doi:10.4049/jimmunol. 1000532 [PubMed: 20639488]

61. Tsuji T, Sabbatini P, Jungbluth AA, et al. Effect of Montanide and poly-ICLC adjuvant on human self/tumor antigen-specific CD4+ T cells in phase I overlapping long peptide vaccine trial. Cancer Immunol Res. 2013;1(5):340-350. doi:10.1158/2326-6066.CIR-13-0089 [PubMed: 24777970]

62. Speiser DE, Liénard D, Rufer N, et al. Rapid and strong human CD8+ T cell responses to vaccination with peptide, I FA, and CpG oligodeoxynucleotide 7909. J Clin Invest. 2005;115(3): 739-746. doi:10.1172/JCI23373 [PubMed: 15696196]

63. Haining WN, Davies J, Kanzler H, et al. CpG oligodeoxynucleotides alter lymphocyte and dendritic cell trafficking in humans. Clin Cancer Res. 2008;14(17):5626-5634. doi: 10.1158/1078-0432.CCR- 08-0526 [PubMed: 18765557]

64. Krieg AM. CpG motifs in bacterial DNA and their immune effects. Annu Rev Immunol. 2002;20:709-760. doi:10.1146/annurev.immunol.20.100301.064842 [PubMed: 11861616]

65. Nava-Parada P, Forni G, Knutson KL, Pease LR, Celis E. Peptide vaccine given with a Toll-like receptor agonist is effective for the treatment and prevention of spontaneous breast tumors. Cancer Res. 2007;67(3):1326-1334. doi:10.1158/0008-5472.CAN-06-3290 [PubMed: 17283170]

66. Peng G, Guo Z, Kiniwa Y, et al. Toll-like receptor 8-mediated reversal of CD4+ regulatory T cell function. Science. 2005;309(5739):1380-1384. doi:10.1126/science.1113401 [PubMed: 16123302]

67. Lee M, Park C-S, Lee Y-R, Im S-A, Song S, Lee C-K. Resiquimod, a TLR7/8 agonist, promotes differentiation of myeloid-derived suppressor cells into macrophages and dendritic cells. Arch Pharm Res. 2014;37(9):1234-1240. doi:10.1007/s12272-014-0379-4 [PubMed: 24748512]

68. Dranoff G, Jaffee E, Lazenby A, et al. Vaccination with irradiated tumor cells engineered to secrete murine granulocyte-macrophage colony-stimulating factor stimulates potent, specific, and longlasting anti-tumor immunity. Proc Natl Acad Sci USA. 1993;90(8):3539-3543. [PubMed: 8097319]

69. Ahlers JD, Dunlop N, Alling DW, Nara PL, Berzofsky JA. Cytokine-in-adjuvant steering of the immune response phenotype to HIV-1 vaccine constructs: granulocyte-macrophage colony- 
stimulating factor and TNF-alpha synergize with IL-12 to enhance induction of cytotoxic T lymphocytes. J Immunol. 1997;158(8):3947-3958. [PubMed: 9103465]

70. Slingluff CL, Petroni GR, Smolkin ME, et al. IMMUNOGENICITY FOR CD8+ AND CD4+ T CELLS OF TWO FORMULATIONS OF AN INCOMPLETE FREUND'S ADJUVANT FOR MULTIPEPTIDE MELANOMA VACCINES. J Immunother. 2010;33(6):630-638. doi:10.1097/ CJI.0b013e3181e311ac [PubMed: 20551833]

71. Faries MB, Hsueh EC, Ye X, Hoban M, Morton DL. Effect of granulocyte/macrophage colonystimulating factor on vaccination with an allogeneic whole-cell melanoma vaccine. Clin Cancer Res. 2009;15(22):7029-7035. doi:10.1158/1078-0432.CCR-09-1540 [PubMed: 19903777]

72. Schwartzentruber DJ, Lawson DH, Richards JM, et al. gp100 peptide vaccine and interleukin-2 in patients with advanced melanoma. N Engl J Med. 2011;364(22):2119-2127. doi:10.1056/ NEJMoa1012863 [PubMed: 21631324]

73. Kirkwood JM, Lee S, Land S, et al. E1696: Final analysis of the clinical and immunological results of a multicenter ECOG phase II trial of multi-epitope peptide vaccination for stage IV melanoma with MART-1 (27-35), gp100 (209-217, 210M), and tyrosinase (368-376, 370D) (MGT) +/IFNa2b and GM-CSF. JCO. 2004;22(14_suppl):7502-7502. doi:10.1200/jco.2004.22.14_suppl. 7502

74. Slingluff CL, Petroni GR, Yamshchikov GV, et al. Immunologic and clinical outcomes of vaccination with a multiepitope melanoma peptide vaccine plus low-dose interleukin-2 administered either concurrently or on a delayed schedule. J Clin Oncol. 2004;22(22):4474-4485. doi:10.1200/JCO.2004.10.212 [PubMed: 15542798]

75. Riemann H, Takao J, Shellman YG, et al. Generation of a prophylactic melanoma vaccine using whole recombinant yeast expressing MART-1. Exp Dermatol. 2007;16(10):814-822. doi: 10.1111/j.1600-0625.2007.00599.x [PubMed: 17845213]

76. Markov OV, Mironova NL, Sennikov SV, Vlassov VV, Zenkova MA. Prophylactic Dendritic CellBased Vaccines Efficiently Inhibit Metastases in Murine Metastatic Melanoma. PLOS ONE. 2015;10(9):e0136911. doi:10.1371/journal.pone.0136911

77. Phua KKL, Staats HF, Leong KW, Nair SK. Intranasal mRNA nanoparticle vaccination induces prophylactic and therapeutic anti-tumor immunity. Scientific Reports. 2014;4:5128. doi:10.1038/ srep05128 [PubMed: 24894817]

78. van Elsas A, Sutmuller RPM, Hurwitz AA, et al. Elucidating the Autoimmune and Antitumor Effector Mechanisms of a Treatment Based on Cytotoxic T Lymphocyte Antigen-4 Blockade in Combination with a B16 Melanoma Vaccine: Comparison of Prophylaxis and Therapy. Journal of Experimental Medicine. 2001;194(4):481-490. doi:10.1084/jem.194.4.481 [PubMed: 11514604]

79. Kooreman NG, Kim Y, de Almeida PE, et al. Autologous iPSC-Based Vaccines Elicit Anti-tumor Responses In Vivo. Cell Stem Cell. 2018;22(4):501-513.e7. doi:10.1016/j.stem.2018.01.016

80. Rosenberg SA, Yang JC, Restifo NP. Cancer immunotherapy: moving beyond current vaccines. Nat Med. 2004;10(9):909-915. doi:10.1038/nm1100 [PubMed: 15340416]

81. Boon T, Coulie PG, Van den Eynde BJ, van der Bruggen P. Human T cell responses against melanoma. Annu Rev Immunol. 2006;24:175-208. doi:10.1146/annurev.immunol. 24.021605.090733 [PubMed: 16551247] 


\section{KEY POINTS}

- $\quad$ Cancer vaccines are formulated with tumor-associated antigens and vaccine adjuvants to elicit a targeted immune response for tumor control.

- $\quad$ The elicited T cell response to tumor-associated antigens may be discordant with tumor control; current strategies of vaccine antigens and adjuvants are under investigation to improve clinical response rates.

- Many clinical trials are currently underway to determine the optimal combinations for the different types of tumor-associated antigens, vaccine adjuvants, and other immunotherapies such as checkpoint blockade therapy. 




Figure 1: Mechanism of immune response induction following vaccination.

Step 1. Vaccination allows tumor antigen to be taken up by antigen-presenting cells (APCs). Step 2. Adjuvant stimulation supports activation and maturation of APCs. Step 3. APCs present antigen to CD8 T cells via MHC class I and to CD4 T cells through MHC class II, resulting in their activation and proliferation and thereby launching an antitumor immune response. 


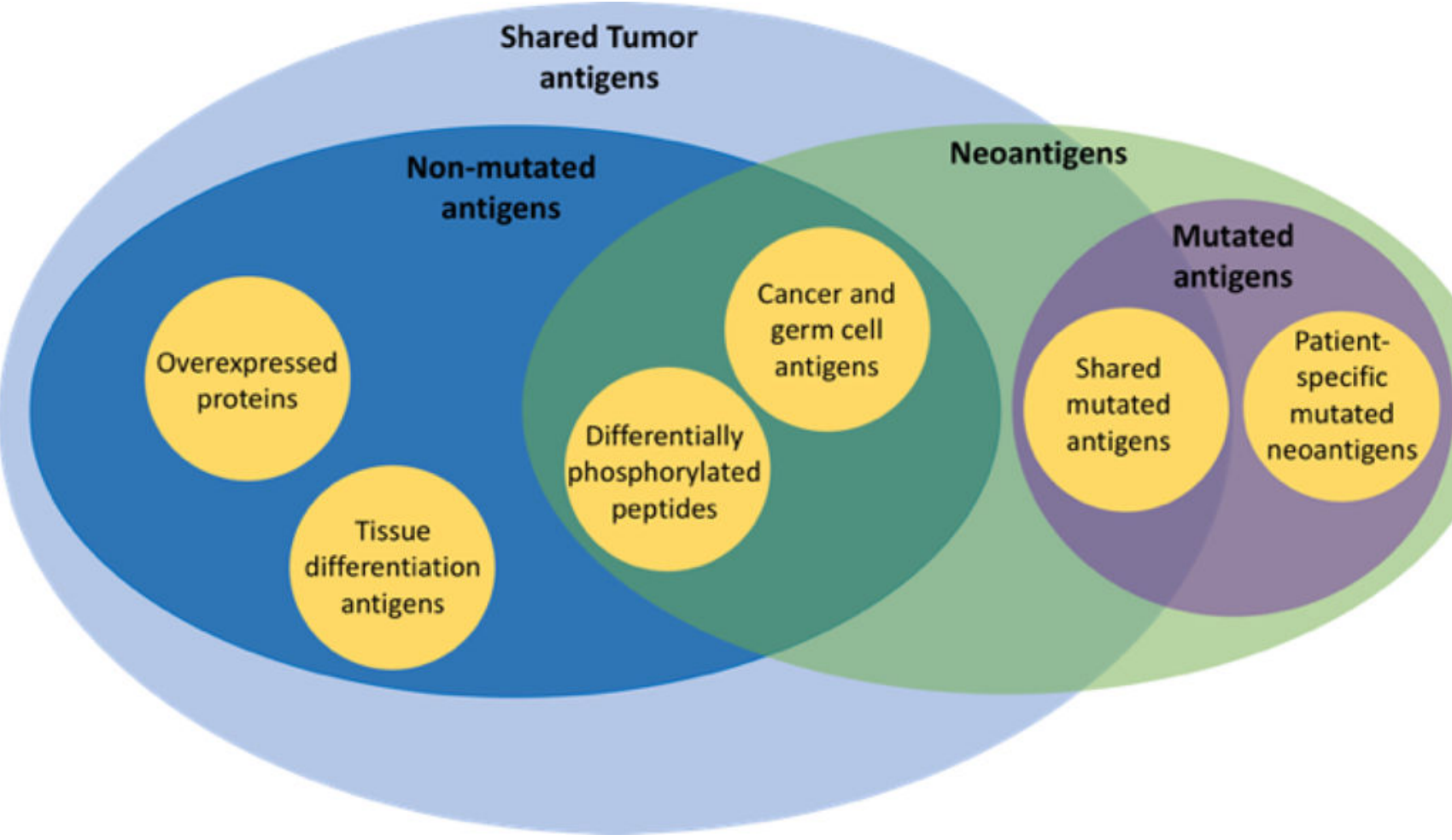

Figure 2:

Different types of tumor-associated antigens in melanoma 
A.

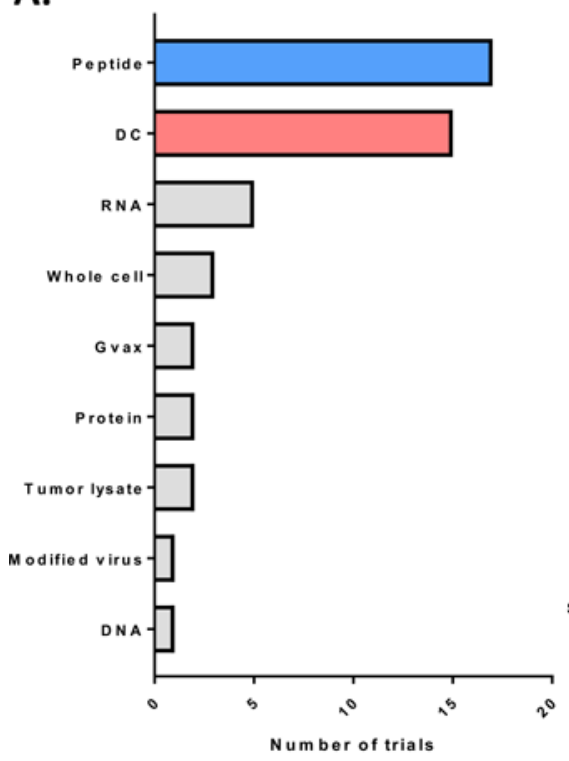

B.

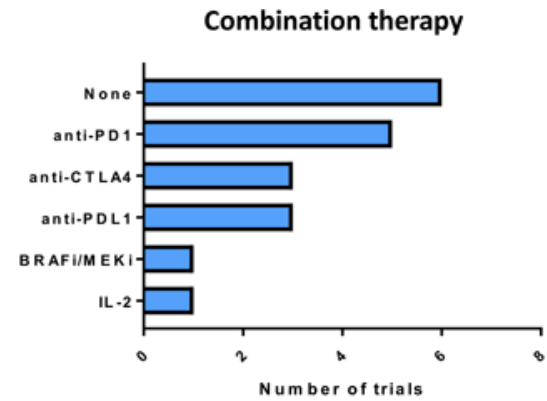

C.



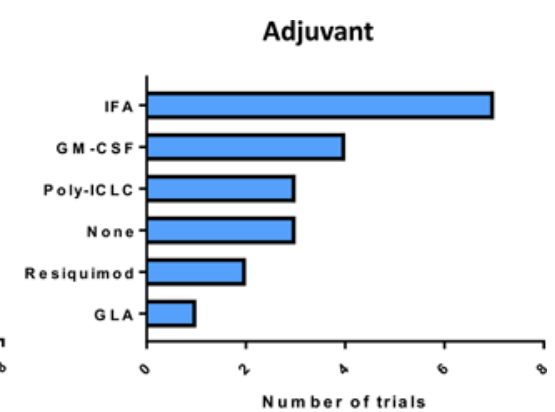

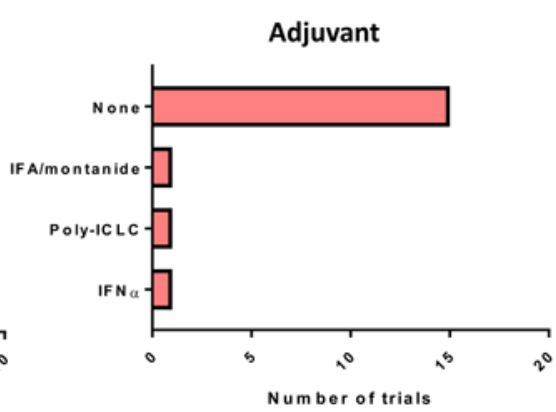

Figure 3: Current vaccine trials in melanoma.

The number of active clinical trials for each melanoma vaccine vehicle as of July 2018 on ClinicalTrials.gov are shown in A. Number of active trials using combination therapies and adjuvants are quantified for peptide vaccines in $\mathbf{B}$ and dendritic cell vaccines in $\mathbf{C}$. 